\title{
EFEITOS DO TREINO DE FLEXIBILIDADE E FORÇA FUNCIONAL NA PRECISÃO EM TESTE DE HANDEBOL
}

Maurício Quian Nogueira

Fabrício Boscolo Del Vecchio

\section{Resumo}

A presente investigação buscou avaliar os efeitos do treinamento funcional resistido (TFR) e de flexibilidade na precisão do arremesso de 9 metros de 10 jogadoras de handebol. Para tal, delineou-se pesquisa quase-experimental, de medidas repetidas, pré-pós intervenção, tendo como estímulos: 30 segundos de alongamento ou 2x10 repetições de pullover na bola. Foram avaliadas: 1) reprodutibilidade, por meio do CCI; 2) consistência interna do teste, a partir do alfa de Cronbach; 3) mudanças no rendimento segundo tipo de intervenção, através de análise de variância. Pontua-se que os treinamentos não proporcionaram mudanças significantes no escore do teste de $9 \mathrm{~m}$; no entanto, $60 \%$ das atletas melhoraram seus resultados com o TFR e 50\% delas pioraram com o treino de flexibilidade.

\section{Palavras-Chave}

Potencialização Pós-Ativação; Flexibilidade; Handebol

\section{EFEITOS DO TREINO DE FLEXIBILIDADE E FORÇA FUNCIONAL NA PRECISÃO EM TESTE DE HANDEBOL}

Maurício Quian Nogueira

Fabrício Boscolo Del Vecchio

\begin{abstract}
The aim of this research was to evaluate effects of functional and flexibility training on accuracy of 9 meters test in 10 handball players. To it, was conducted quasi-experimental counter-balanced experiment, with repeated measurements, with stimulus: $30 \mathrm{~s}$ of stretching exercises or $2 \times 10$ repetitons of pullover with ball. Was evaluated: 1) reproducibility, by ICC; 2) Internal consistency, with Cronbach alfa; 3) modifications in performance, with anova oneway. Different training methods didn't modify 9 meters score. Meanwhile, $60 \%$ of athletes improver her results with functional training and $50 \%$ decrease with flexibility training.
\end{abstract}

\section{Key-Words}

Potentiation Post-Activation; Flexibility; Handball. 


\section{INTRODUÇÃO}

$\mathrm{O}$ processo de treinamento físico tem como características elevar o rendimento desportivo de atletas das mais variadas modalidades. Expressa-se de modo crônico, a partir do acúmulo de diversas sessões e, também, de forma aguda, exibindo efeitos de curto prazo, em geral, subseqüentes às sessões cumpridas (OZOLIN, 1989, WEINECK, 1999).

Dentre as manifestações agudas, estão aquelas decorrentes dos estímulos de alongamento e de força muscular. Quanto ao primeiro, estudos como de Pope et al. (2000) verificam que essa atividade realizada antes do exercício principal não reduz significativamente o risco de lesão, além de observarem diminuição significativa no desempenho da força. Arruda et al. (2006), por exemplo, concluíram que exercícios de alongamento estático, executados antes do teste de 10 Repetições Máximas (RM), na máquina de supino reto, provocam queda no número de repetições completadas.

Aparentemente, estímulos de flexibilidade tendem a prejudicar desempenhos que envolvam potência muscular, no entanto, em atividades relativamente simples, como o salto vertical e no arremesso de medicinebol (NELSON et al., 2005). Por outro lado, não foram registradas mudanças na amplitude e rapidez dos membros inferiores durante a execução de chutes entre futebolistas e nem modificações na velocidade de deslocamento da bola no saque de tenistas (YOUNG et al., 2004, KNUDSON et al., 2004). Ou seja, em situações mais completas o treino de flexibilidade não tende a prejudicar o rendimento desportivo.

Por sua vez, o treino de força muscular conduz a incrementos significativos em tarefas simples e complexas. Para esta manifestação, dá-se o nome de Potencialização Pós-Ativação. Segundo Baker (2003), existem ganhos em atuações multi-articulares, como no supino reto, com séries de seis repetições, a $65 \%$ da carga máxima (CM). São relatadas, ainda, decorrências potencializadoras de exercícios pliométricos simples e combinados com pesos elevados (MASAMOTO et al., 2003, EBBEN, 2002, DUTHIE; YOUNG; AITKEN, 2002).

Um modo de treinamento de força que vem ganhando espaço nas práticas desportivas constitui-se pelo treinamento funcional resistido - TFR (CAMPOS; CORAUCCI, 2004). Ele visa ao aprimoramento da capacidade funcional do corpo humano, está baseado na melhoria dos aspectos neuro-musculares, especialmente o proprioceptivo e o sensório motor. Quanto a este último, algumas evidências indicam que ele pode aumentar a atividade eletromiográfica e a taxa de desenvolvimento de força após 4 
semanas de treinamento (GRUBER et al., 2007); no entanto, não são conhecidos estudos que abordam sua aplicação aguda.

Este tipo de trabalho auxilia os músculos agonistas e antagonistas a estabilizarem o complexo articular ativamente, sendo que o aumento na taxa de desenvolvimento de força é conseqüência do aumento da sensitividade do reflexo monosináptico de alongamento (FRANCHINI; DEL VECCHIO, 2007). Assim, o sistema neuromuscular é capaz de ativar os músculos mais eficientemente no início do desenvolvimento de força. Do ponto de vista funcional, isso é importante para estabilizar o complexo articular em situações de instabilidade (GALLHOFER, 2003).

Especificamente quanto ao Handebol, a estabilidade corporal é fundamental no processo de arremesso da bola ao gol. Como os praticantes devem arremessá-la de modo que o goleiro não a alcance, são desejadas características de potência muscular e precisão. Como a falta faz parte da tática defensiva e toda infração é cobrada na linha tracejada (ou linha dos 9 metros), jogadores mais aptos neste tipo de habilidade podem, eventualmente, gerar maior número de gols para suas equipes.

Assim, este trabalho tem como objetivo investigar os efeitos de exercícios prévios de flexibilidade e de força funcional, na precisão de jogadoras de Handebol durante o arremesso de 9 metros com apoio.

\section{MATERIAIS E MÉTODOS}

\section{Tipo de Estudo e Caracterização das Variáveis}

De cunho quantitativo, caracteriza-se como quase-experimental de medidas repetidas (DAWSON; TRAPP, 2003). Como variável dependente tomou-se o escore produzido no teste de $9 \mathrm{~m}$ de handebol, já as independentes foram aquelas relacionadas ao treinamento de flexibilidade e de força.

\section{GRUPO PESQUISADO}

Grupo composto de dez atletas do sexo feminino, semi-profissionais do time de handebol da cidade de Campinas, apoiado pela prefeitura da cidade e pela Faculdade Metropolitana de Campinas - Metrocamp e FIEC - Fundo de Investimentos Esportivos de Campinas. A pesquisa foi realizada no Complexo Esportivo Rogê Ferreira, no período da tarde em dois dias não subseqüentes.

\section{DELINEAMENTO DA PESQUISA E INSTRUMENTOS DE AVALIAÇÃO}

$\mathrm{Na}$ condução da investigação, foram utilizados dois dias e três procedimentos. O primeiro, situação controle, o segundo e terceiro, de flexibilidade ou de força funcional, determinados de forma contra- 
balanceada, realizadas no mesmo dia com 45 minutos de intervalo entre elas. Para os três momentos, as atletas realizaram procedimento inicial (PI) de aquecimento por três minutos em forma de trote moderado em volta da quadra.

O instrumento de avaliação utilizado neste trabalho foi o Teste de arremesso de Handebol da linha dos 9m de Zinn (TRITSCHLER, 2003). Este teste tem como objetivo avaliar a habilidade no arremesso frontal para a marcação dos gols no Handebol, conta com diversos setores entre as três traves (Figura 1) e somatório de 5 arremessos, válidos segundo local de entrada no gol, desde que não ocorra quique da bola.

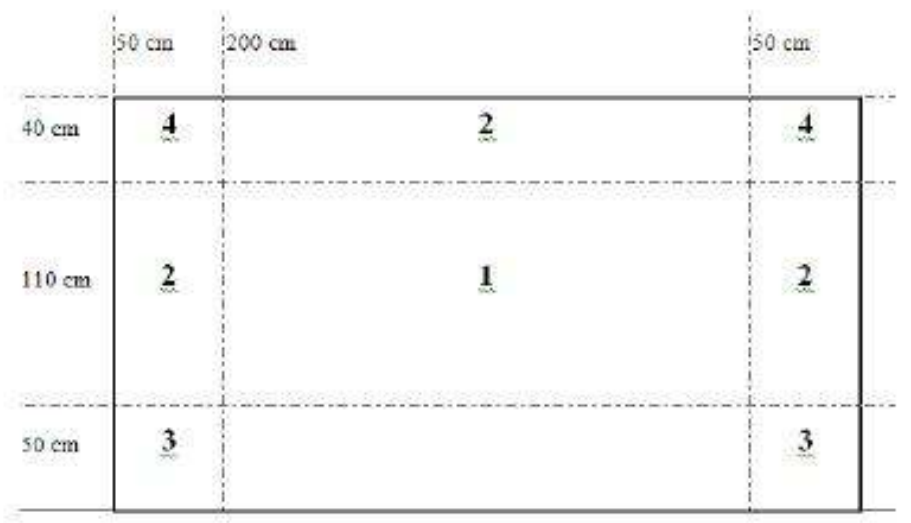

Figura 1. Tamanho das traves no handebol e divisão setorial com respectivos pontos

Na situação:

a) controle (PIC): as atletas realizaram o teste inicial (PIC-A), repousaram por três minutos e foram avaliadas novamente (PIC-D).

b) flexibilidade (PIF): após o primeiro teste (PIF-A), executaram-se 2 séries de 30 segundos cada de alongamentos estáticos, com 1 minuto de intervalo entre as séries. Durante estes trinta segundos, três grupos musculares, tríceps, peitoral e grande dorsal, foram escolhidos, pois estão diretamente ligados ao movimento de arremesso do handebol. Cada grupo muscular teve dez segundos de estímulo.

Depois da ultima série ser executada, a atleta ficou em repouso por três minutos, e após este intervalo executou mais cinco arremessos com apoio da linha de nove metros (PIF-D).

c) força funcional (PIR): Após PI e o teste de 9 metros (PIR-A) serem realizados, a atleta 
executou 2 séries de 10 repetição do exercício de força funcional pullover na bola, com carga padronizada de $10 \mathrm{~kg}$ e intervalo de um minuto entre as séries. Após completarem as duas séries de dez repetições cada, com um minuto de intervalo entre as séries, ocorreu intervalo em repouso absoluto de três minutos, para depois executarem mais cinco arremessos com apoio da linha de nove metros (PIR-D).

\section{ANÁLISE ESTATÍSTICA}

Os dados são apresentados segundo medidas de centralidade e dispersão. Para verificação da consistência interna do teste, aplicaram-se:

i) o teste de alfa de Cronbach na situação PIC-A e,

ii) teste $t$ de Student para amostras pareadas (entre PIC-A e PIC-B) e, para a reprodutibilidade,

iii) o coeficiente de correlação intraclasse (CCI) (THOMAS; NELSON, 2002).

Após verificação da normalidade dos dados (prova de Shapiro-Wilk), conduziu-se análise de variância one-way com post-hoc de Scheffé. Adotou-se $\mathrm{p}<0,05$ como representante de diferenças significantes.

Adicionalmente, os valores são apresentados segundo três categorias de rendimento: piora, manutenção ou melhora.

\section{RESULTADOS}

Primeiramente, quanto às propriedades psicométricas, não foram registradas diferenças significantes nas situações PIC-A e PIC-D, apontando adequada estabilidade, observa-se boa consistência interna (alfa de Cronbach $=0,90$ ) e alta reprodutibilidade (medida entre PIC-A e PIF-A, CCI = 0,78, p<0,05).

As medidas descritivas são apresentadas na Tabela 1. Não foram observadas diferenças significantes entre quaisquer situações registradas. Pontua-se, apenas, que houve tendência à melhora nas três situações: controle, flexibilidade e força funcional.

Consideraram-se também as categorias de rendimento (melhora, piora e manutenção), pois, desta forma, não se pondera o quanto ocorreu de melhora, mas apenas se ela existiu ou não, assim como a perda de desempenho. 
TABELA 1- Medidas descritivas, segundo momento e intervenção

\begin{tabular}{|c|c|c|c|c|c|c|c|}
\hline & Média & $\begin{array}{l}\text { Desvio } \\
\text { padrão }\end{array}$ & $\begin{array}{c}\text { Erro } \\
\text { Padrão }\end{array}$ & $\begin{array}{r}95^{\circ} \\
(\mathrm{min}\end{array}$ & $\begin{array}{l}\text { IC } \\
\operatorname{nax})\end{array}$ & Mínimo & Máximo \\
\hline$\overline{P I C-A}$ & 7.30 & 5.38 & 1.70 & 3.45 & 11.15 & 0.00 & 16.00 \\
\hline PIC-D & 9.30 & 3.47 & 1.10 & 6.82 & 11.78 & 6.00 & 15.00 \\
\hline PIF-A & 7.70 & 4.60 & 1.45 & 4.41 & 10.99 & 1.00 & 16.00 \\
\hline PIF-D & 8.30 & 2.95 & 0.93 & 6.19 & 10.41 & 3.00 & 12.00 \\
\hline PIR-A & 7.10 & 3.60 & 1.14 & 4.52 & 9.68 & 3.00 & 12.00 \\
\hline PIR-D & 8.80 & 3.70 & 1.17 & 6.14 & 11.45 & 4.00 & 16.00 \\
\hline
\end{tabular}

PIC-A: teste de 9m, na situação controle, antes; PIC-D: teste de 9m, na situação controle, depois; PIF-A: teste de 9m, na situação flexibilidade, antes; PIF-D: teste de 9m, na situação flexibilidade, depois; PIRA: teste de 9m, na situação funcional, antes; PIR-D: teste de 9m, na situação funcional, depois.

Para tal, subtraiu-se o valor do pós-intervenção (depois) daquele da pré-intervenção (antes) de cada um dos momentos. Registra-se que o Treinamento Funcional Resistido gerou maior número de melhoras e o treino de Flexibilidade, maior quantidade de pioras, na relação antes-depois (Quadro 1).

QUADRO 1. Variação categórica no rendimento, segundo tipo de intervenção.

\begin{tabular}{lccc}
\hline Mudança & Controle & Flexibilidade & Força \\
\hline Melhorou & 6 & 3 & 6 \\
Piorou & 4 & 5 & 2 \\
Manteve & 0 & 2 & 2 \\
\hline Total & 10 & 10 & 10 \\
\hline
\end{tabular}

Embora a literatura apresente:

1) Benefícios do treinamento funcional e

2) Prejuízos decorrentes do treinamento de flexibilidade, estes comportamentos não foram identificados de forma significante no presente estudo.

Isto se deve, possivelmente, a: 
1) Falta de prática de treinamento de força entre as atletas, pois as mesmas não tinham vivências significativas com treinamento funcional ou mesmo musculação e,

2) Tempo de intervenção para o alongamento relativamente baixo, pois foram apenas 10 segundos de estímulos por grupo muscular.

Quanto à primeira hipótese, existem algumas evidências de que pessoas podem ser mais ou menos responsivas à potencialização pós-ativação, mesmo que feita sob perspectiva do treinamento funcional, tanto pela experiência em treinamento com pesos, quanto pela individualidade biológica (SMITH; FRY, 2007).

Estudos que não identificaram modificações no desempenho, decorrente de estímulos de força, ou de alongamento, apontam que o tempo de intervenção pode ser fator determinante. Investigações com lutadores observaram que o treino de flexibilidade não prejudicou o rendimento dos atletas, possivelmente devido à duração do estímulo (MIARKA; DEL VECCHIO, 2007, DEL VECCHIO; PALERMO JR. 2007). Na presente investigação este fator também pode estar presente, pois cada grupamento muscular (Peitoral, Tríceps e Grande Dorsal) foi alongado por apenas 10 segundos.

Já quanto ao treino funcional resistido, embora tenha provocado maior quantidade de melhoras, quando se toma o escore como resultado, não foram apresentadas de forma significante. Talvez, por envolver características como:

1) senso de posição,

2) senso de movimento, e;

3) senso de força (FRANCHINI; DEL VECCHIO, 2007), talvez seja necessária maior quantidade de treinos para a gênese das melhoras. Isto, em parte, pode ser comprovado pelo estudo de Gruber et al., (2007), o qual teve duração de 4 semanas, com sessões 3 vezes na semana.

No entanto, quando consideradas somente as categorias de mudança -melhora, manutenção e perda pontua-se que o Treinamento de Força Funcional tendeu a apresentar maior percentual de melhora que na situação precedente de flexibilidade. Ou seja, aparentemente a magnitude do aumento pode ter sido baixa, mas o incremento esteve presente em $60 \%$ das atletas. Por outro lado, o treinamento de flexibilidade piorou o resultado no teste de precisão em $50 \%$ delas. 
Dessa forma, novos estudos têm de ser conduzidos tomando os seguintes cuidados:

1) número maior de unidades observacionais;

2) maior tempo no treino de flexibilidade e,

3) ajuste da carga, no treinamento de força, em função da capacidade de cada atleta.

\section{CONCLUSÕES}

Os estímulos conduzidos na presente investigação não modificaram de forma significante a precisão no teste de $9 \mathrm{~m}$ do handebol. No entanto, de forma categórica, o treinamento funcional resistido proporcionou melhora para $60 \%$ das atletas, ao passo que o de flexibilidade prejudicou o desempenho de metade delas.

\section{REFERÊNCIAS}

ARRUDA, F. L. B., et al. A influência do alongamento no rendimento do treinamento de força. Revista Treinamento Desportivo, Curitiba, v. 7, n.1, p. 01-05, jan. 2006.

BAKER, D. Acute effect of alternating heavy and light resistances on power output during upper-body complex power training. Journal of Strength and Conditioning Research, Colorado Springs, v.17, n.3, p.493-497, aug, 2003.

CAMPOS, M. A.; CORAUCCI NETO, B. Treinamento funcional resistido. Rio de Janeiro: Revinter, 2004.

DAWSON, B; TRAPP, R.G. Bioestatística básica e clínica. 3. ed Rio de Janeiro: McGraw-Hill do Brasil, 2003.

DUTHIE, G. M.; YOUNG, W. B.; AITKEN, D. A. The acute effects of heavy loads on jump squat performance: an evaluation of the complex and contrast methods of power development. Journal of Strength and Conditioning Research, Colorado Springs, v.16, n. 4, p. 530-538, nov. 2002.

EBBEN, W.P. Complex training: a brief review. Journal of Sports Science and Medicine, Bursa, v.1, n.1, p. 42-46, jun. 2002.

FRANCHINI, E.; DEL VECCHIO, F. B. Preparação física para atletas de judô. São Paulo: Phorte, 2007.

GALLHOFER, A. Proprioceptive training: considerations for strength and power production. In: KOMI, P.V. Strength and power in sport. Oxford: Blackwell Science: 2003, p. 331-342.

GRUBER, M.; GRUBER, S.B.H.; TAUBE, W. et al. Differencial effects of ballistic versus sensorimotor CONEXÕES, revista da Faculdade de Educação Física da UNICAMP, Campinas, v. 6, ed. especial, p. 111- , jul. 2008. 
training on rate of force development and neural activation in humans. Journal of Strength and Conditioning Research, Colorado Springs, v. 21, n.1, p. 274-282, feb. 2007.

KNUDSON, D. V.; NOFFAL, G. J.; BAHAMONDE, R. E.; BAUER, J. A.; BLACKWELL, J. R. Stretching has no effect on tennis serve performance. Journal of Strength and Conditioning Research, Colorado Springs, v.18, n. 3, p. 654-656, aug. 2004.

MASAMOTO, N.; LARSON, R.; GATES, T.; FAIGENBAUM, A. Acute effects of plyometric exercise on maximum squat performance in male athletes. Journal of Strength and Conditioning Research, Colorado Springs, v.17, n.1, p. 68-71, feb. 2003.

MIARKA, B.; DEL VECCHIO, F. B. Efeitos do treinamento físico agudo no potencial pós-ativação de força e alongamento muscular entre judocas. In: CONGRESSO INTERNACIONAL DE CIÊNCIAS DO ESPORTE, 2. 2007, Recife. Anais... Recife, 2007. p. 277.

NELSON, A. G.; DRISCOLL, N. M.; LANDIN, D. K.; YOUNG, M. A.; SCHEXNAYDER, I. C. Acute effects of passive muscle stretching on sprint performance. Journal of Sports Science, Oxford, v. 23, n. 5, p. 449-454, may. 2005.

OZOLIN, N. G. Sistema contemporáneo de entrenamiento deportivo. Habana: Editorial CientíficoTécnica, 1989.

POPE, R.; HERBERT, R.; KIRWAN, J. D.; GRAHAM, B. J. A randomized trial of preexercise stretching for prevenion of lower-limb injury. Medicine and Science in Sports and Exercise, Baltimore, v. 32, n.2, p. 271-277, feb. 2000.

SMITH, J. C.; FRY, A. C. Effects of a ten-second maximum voluntary contraction on regulatory myosin ligth-chain phosphorylation and dynamic performance measures. Journal of Strength and Conditioning Research, Colorado Springs, v. 21, n. p. 73-76, feb. 2007.

THOMAS, J. R.; NESLON, J. K. Métodos de pesquisa em atividade física. 3. ed. Porto Alegre: ArtMed, 2002.

TRITSCHLER, K. Medida e avaliação em Educação Física e Esportes de Barrow \& McGee. 5. ed. Barueri: Manole, 2003.

WEINECK, J. Treinamento ideal. Barueri: Manole, 1999.

YOUNG, W.; CLOTHIER, P.; OTAGO, L.; BRUCE, L.; LIDDELL D. Acute effects of static stretching on hip flexor and quadriceps flexibility, range of motion and foot speed in kicking a football. Journal of Science and Medicine in Sport, v. 7, n. 1, p. 23-31, mar. 2004. 
Maurício Quian Nogueira

Faculdades Integradas Metropolitanas de Campinas

\section{Fabrício Boscolo Del Vecchio}

Faculdades Integradas Metropolitanas de Campinas

Faculdade de Educação Física - UNICAMP

Referência do artigo

\section{ABNT}

NOGUEIRA, M. Q.; DEL VECCHIO F. B. Efeitos do treino de flexibilidade e força funcional na precisão em teste de handebol. Conexões, v. 6, ed. especial, p. 122-131, 2008.

\section{APA}

Nogueira MQ, \& DEL VECCHIO FB. (2008). Efeitos do treino de flexibilidade e força funcional na precisão em teste de handebol. Conexões, v. 6 (ed. especial), 122-131.

\section{VANCOUVER}

Nogueira MQ, DEL VECCHIO FB. Efeitos do treino de flexibilidade e força funcional na precisão em teste de handebol. Conexões, 2008; 6 (ed. especial): 122-131. 